\title{
Jacobian Formulation for Two Classes of Cooperative Continuum Robots
}

\author{
Somayeh Norouzi Ghazbi ${ }^{1}$, Ali Mehrkish ${ }^{2}$, Farrokh Janabi-Sharifi ${ }^{3 *}$ \\ ${ }^{1,2,3}$ Mechanical and Industrial Engineering Department, Ryerson University, Toronto, Canada. \\ *fsharifi@ryerson.ca
}

\begin{abstract}
Continuum robots (CRs) are an important class of robots inspired by the biological counterparts. Recently, a new paradigm of using multiple CRs, under cooperative continuum robots (CCRs), has been introduced to increase the performance of CRs. The motivation behind this work is to provide a detailed discussion on the concept of CCRs and a detailed description of its two different sub-configurations, including Co-manipulative and Target-assist CCRs. This paper also presents kinematic modeling and Jacobian derivation of these two sub-configurations. It has been shown that kinematics of Co-manipulative CCRs could be well estimated using the DH approach; while, for deriving kinematics of the second subconfiguration, a combination of DH approach and an exact kinematic modeling approach, using Cosserat rod theory, has to be used. The obtained Jacobians could be used for control purposes and CR-based grasp synthesis.
\end{abstract}

Keywords-Continuum robots; cooperative continuum robots; kinematics; Jacobian; grasp synthesis

\section{INTRODUCTION}

In recent years, design, modeling, and control of Continuum Robots (CRs) have gained significant research attention [1]-[6]. Their applications have been on the rise due to their continuum backbone structure. Continuum backbone enables CRs to operate in constrained spaces [7] and makes them a safer choice for interacting with humans. Various designs of CRs are heavily influenced by the research on natural continuum structures such as elephant's trunks [8], snakes, or octopus's tentacles [9]. The development of new natural-based structures of CRs and introducing new configurations of them are still among active research areas in robotics [10]-[13]. Until now, a couple of configurations have been introduced, including single-CRs [14], parallel-CRs [15], Series-CRs [16], and Cooperative-CRs [4].

Single-CRs often include a couple of tendons, each actuated by a separate actuator that has continuous interaction with a support backbone [14]. Series-CRs consist of a couple of segments arranged in series such that each segment has a separate actuation system [17]. Shortcomings of single- and series-CRs include their low payload, limited degrees of actuation (leading to poor tip degrees-of-freedom), questionable maneuverability, poor accuracy, and poor tip stiffness. ParallelCRs [18]-[20] have been proposed with some potential for addressing some of the above shortcomings of individual CRs.
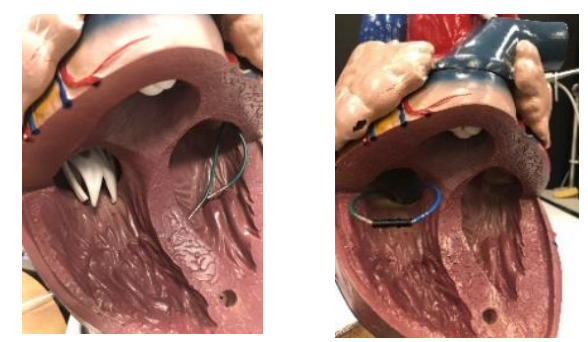

Figure 1. Different configurations of CCRs in medical applications

They consist of either a few CRs arranged in parallel to form closed kinematic chains or a couple of segments sharing a common base position. Parallel-CRs provide increased precision [21] and larger torsional stiffness [22]. They also make it possible to form complex cross-sections, which consequently result in boosted performance in doing more complicated tasks [23]. Another alternative is cooperative-CRs (CCRs) [4] as shown in Fig. 1. CCRs consist of a set of CRs providing degreesof-freedoms over the base positions of the involved CRs, and their contact points. Regarding the contact points, both position and orientation in which one CR reaches the other/s could be variable during the defined operative task. The advantages of incorporating cooperative configurations over the use of series or parallel sets are enlarged workspace and improved manipulability of the coupled system. Another advantage of CCRs is their adaptive configurations enabling them to pass through complicated structures. They provide better control over tooltips of target CRs and increase the total stiffness of the tooltip, which enables applying larger forces.

One of the applications of CCRs is in minimally invasive surgeries. For instance, in the treatment of ventricular tachycardia, a cooperative configuration allows multiple entrypoints to facilitate access and mating of coupling catheters that could be difficult or can cause unnecessary damages to tissues when the single-entry point is selected for a few catheters. It is interesting to note that in operation on the left ventricle, punctures are usually made to access from the right ventricle, and if different entry points are made available, such punctures could be minimized. In addition to providing better control over tooltips of target CRs, cooperative sets also increase their stiffness allowing larger force applications that are important for the success of many interventional procedures. 
This paper presents full-kinematics modeling and derivation of Jacobian formulation of two main sub-configurations of CCRs, i.e., (1) Target-assist cooperative CCRs and (2) Comanipulative CCRs. The two sub-configurations are shown in Fig. 1.

In this paper, as our first contribution, we extend some of the ideas in [4] to full-kinematic modeling of Target-assist CCRs. The model developed in our previous paper considers a special case of cooperation in which the Target-CR is bent first, and then the cooperation is formed. That model does not count the simultaneous bending actuation of the Target- and Assist-CR. In this paper, we expanded the model so that both Assist- and Target-CR can have simultaneous actuation. Moreover, to increase the model simplicity and still not sacrificing the accuracy, the sub-configuration is considered as two separate parts, and a combination of DH-based and Cosserat-based are utilized to obtain the full-model.

The second contribution of this paper includes kinematic modeling and Jacobian derivation of the Co-manipulative CCR. It is for the first time that the full model, which includes both the involved CR arms and the grasped object, is derived for such configuration.

For both parts, the result Jacobians have reasonable timecost, making them appropriate for a wide range of applications from real-time control to grasp synthesis. Part II defines the concepts of both Co-manipulative and Target-assist subconfigurations. Parts III and IV present forward kinematics and Jacobian derivation of Co-manipulative and Target-assist CCRs, respectively. Simulations are presented in Part V, and finally, conclusion is presented in Part VI.

\section{COOPERATIVE CONTINUUM ROBOTS: CONCEPT AND CLASSIFICATIONS}

The minimum number of $\mathrm{CRs}$ required to form a $\mathrm{CCR}$ configuration is two. According to the defined task for CCRs, different selections could be made on the relative base positions and relative tip positions of the involved CRs, which ends up in different sub-configurations of CCRs.

Two main such sub-configurations are shown in Fig. 2. We name them Target-assist CCR and Co-manipulative CCR.

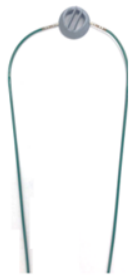

(a)

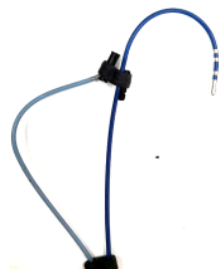

(b)
Figure 2. Two main sub-configurations of CCRs: (a) Target-assist-CCR, (b): Co-manipulative CCR

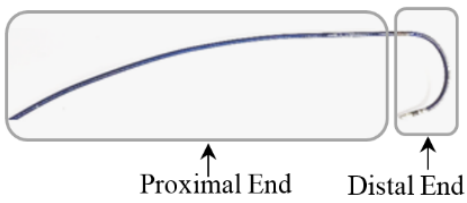

Figure 3. Breakdown of the CRs in Figure (2) to its distal and proximal ends
In this paper, the involved CRs are selected to be of a common kind of CRs used in minimally invasive surgeries. Bodies of these CRs two different internal mechanisms for their proximal- and distal-ends (Fig. 3). The proximal-end is not actuable, and its main functionality is to enable remote actuation of the distal-end. On the contrary, distal end is internally actuated. A set of tendons connected to a rotary knob from one end and fixed to the distal-end-plate from the other end, actuate the distal-end. The distal-end can easily bend in response to the internal actuation. Also similar to the other internally-actuated CRs, the distal-end shows stiffness in response to external forces and moments.

The sub-configuration of Co-manipulative CCRs (shown in Fig. 2.a), consists of a dual/or multiple CRs which act in parallel on the object. The forward kinematic model and Jacobian of Comanipulative CCR is a function of the object geometry, contact type, positions of contact points on the object, and the postures of the involved CRs.

Co-manipulative CCRs allow for the grasp and manipulation of objects, which are a significant domain of CRs application. This application is specially very beneficial in minimally invasive surgeries (MISs). For instance, some possible applications include taking tissue samples from internal organs and pick and placement of the mitral valve during valve replacement.

The sub-configuration of Target-assist CCR consists of a target CR and one/a set of assistive CRs. In this subconfiguration (Fig. 2.b), CRs are located back-to-back. In such an arrangement, the bending motion of the distal end of the Assist-CR is almost blocked by the proximal end of target-CR. Therefore, the only left motion for the Assist-CR is insertion into the space between the contact point and the base. As shown in Fig. 2.a and 2.b, inserting of a bigger portion of the Assist-CR pushes the contact point downward.

\section{CO-MANIPULATIVE CCRS}

In this part, a planar symmetric configuration consisting of two CRs with the same actuation mechanism is considered for the modeling purposes (Fig. 4).

Each of the involved CRs is considered as a single serial chain. The Co-manipulative CCR shown below includes one closed kinematic chain formed by two single serial chains and the object.

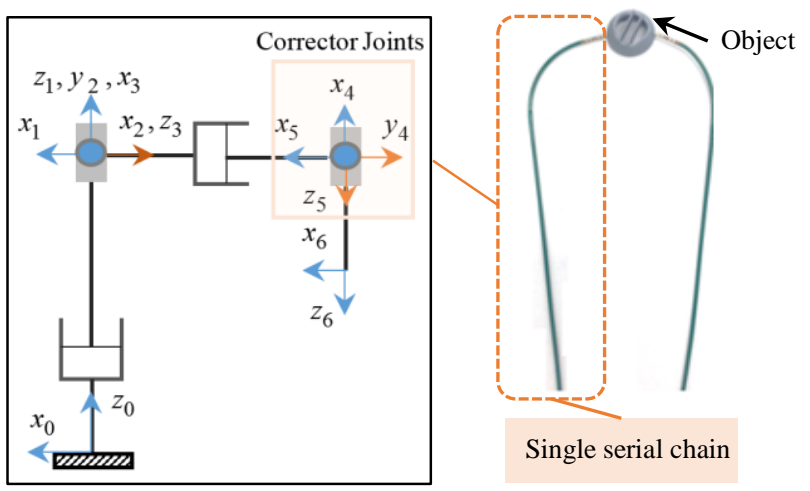

Figure 4. Co-manipulative CCR and the assigned coordinate frames 
The forward kinematic and full Jacobian of the model could be obtained within three steps:

Step I: Jacobian derivation of the single serial chains

For the $i$-th serial chain, $\mathbf{J}_{i}$ represents the $i$-th arm configuration and relates the robot end-effector velocities, $\mathbf{v}_{C-\text { rob }}^{i}$, to the actuator generalized velocities $\dot{\mathbf{q}}_{i}$ through

$$
\mathbf{v}_{C-r o b}^{i}=\mathbf{J}_{i} \dot{\mathbf{q}}_{i},
$$

Step II: Grasp matrix calculation [24]

The Grasp matrix, $\mathbf{G}_{i}$, represents the object geometry and maps the velocities of the grasped object at the contact points, $\mathbf{v}_{C-o b j}^{i}$, to the velocities, $\mathbf{t}$, of the grasped object at any arbitrary point through (2),

$$
\mathbf{v}_{C-o b j}^{i}=\mathbf{G}_{i}^{T} \mathbf{t} .
$$

Hence, the relative velocities between the object and the robot at each contact point are obtained as (3),

$$
\mathbf{v}_{C-o b j}^{i}-\mathbf{v}_{C-r o b}^{i}=\left(\mathbf{J}_{i}-\mathbf{G}_{i}{ }^{T}\right)\left(\begin{array}{c}
\dot{\mathbf{q}}_{i} \\
\mathbf{t}
\end{array}\right) .
$$

Step III: To form the selection matrix according to the contact types [24]

Selection matrices, $\mathbf{B}_{i}$, are used to define contact types, i.e., they chose which components of contact forces and moments and contact linear and rotational velocities transmit between the robot and the object and apply constraints to the problem. Equation (4),

$$
\mathbf{B}_{i}\left(\mathbf{v}_{C-o b j}^{i}-\mathbf{v}_{C-r o b}^{i}\right)=\mathbf{0},
$$

defines the contact constrain equation for the $i_{\text {th }}$ contact. To write the kinematics of co-manipulative CCRs more compactly, we block-diagonalize all the matrices and stack all the vectors to present kinematics relationship as

$$
\mathbf{B}\left(\mathbf{J}-\mathbf{G}^{T}\right)\left(\begin{array}{c}
\dot{\mathbf{q}} \\
\mathbf{t}
\end{array}\right)=\mathbf{0} .
$$

Finally, the transformation from the actuator velocities to the velocities of the grasped object, which is commonly named as grasp Jacobean matrix, $\mathbf{H}$, obtained as,

$$
\begin{aligned}
& \mathbf{t}=\mathbf{H} \dot{\mathbf{q}}, \\
& \mathbf{H}=\left[\left(\mathbf{B G}^{T}\right)^{+}(\mathbf{B J})\right],
\end{aligned}
$$

where (.) $)^{+}$is pseudoinverse.

In (6), the grasp matrix and the selection matrix are defined and obtained according to the object geometry, contact poses, and types. Therefore, considering a specific $\mathbf{G}$ and contactmodel, $\mathbf{J}_{i}$ for the involved CRs needs to be derived to get the full Jacobian of the Co-manipulative sub-configuration.

Constants curvature [10] and Cosserat rod [7] are two commonly used approaches in kinematic modeling of CRs. Constant curvature approach shows promises when a CR is operating in the absence of external forces and moments. On the other hand, the Cosserat rod can obtain an exact model of CRs even if forces and moments are applied. In modeling of CRs, the preference is to use constant curvature since Cosserat rod is time-expensive due to ending up in a set of ordinary differential equations.

In the co-manipulative sub-configuration, interaction forces present at contact points acting as external forces and moments applied to the tip of the involved CRs. However, there are some justifications that allow us to utilize constant curvature approach here including

(1) In problems of manipulating light-weight objects, the interaction forces are not as significant as the internally applied actuation, meaning that their effect on the deformation of the CR could be neglected.

(2) If the entire manipulation breaks down into the pre-grasp problem, it could be considered that in the grasp problem, interaction forces do not appear. Also, in the object manipulation, the model uncertainties due to making constant curvature assumption could be compensated by applying a robust controller.

Therefore, in this part, the involved CRs, a closed-form Jacobian based on constant curvature is derived. According to the physical model and the assigned DH parameters shown in Fig. 4, the modified $\mathrm{DH}$ table is illustrated in Table I, respectively.

\begin{tabular}{cccccccccc}
\hline \multicolumn{1}{c}{ TABLE I: MODIFIED D-H TABLE } \\
\hline \hline Link & $a$ & $\alpha$ & $d$ & $\theta$ & Link & $a$ & $\alpha$ & $d$ & $\theta$ \\
\hline \hline 1 & 0 & 0 & $d_{1}$ & 0 & 4 & 0 & $\frac{\pi}{2}$ & $d_{4}$ & 0 \\
2 & 0 & $\frac{\pi}{2}$ & 0 & $\pi+\theta_{2}$ & 5 & 0 & $\frac{\pi}{2}$ & 0 & $3 \frac{\pi}{2}+\theta_{5}$ \\
3 & 0 & $\frac{\pi}{2}$ & 0 & $\frac{\pi}{2}+\theta_{3}$ & 6 & 0 & 0 & $d_{7}$ & 0 \\
\hline \hline
\end{tabular}

According to Table I, the forward kinematics, $\mathbf{A}$, and Jacobian of the CR, $\mathbf{J}_{i}$, can be obtained as,

$$
\begin{aligned}
& \mathbf{A}=\left[\begin{array}{cccc}
\mathrm{c}\left(\theta_{2}\right) \mathrm{c}\left(2 \theta_{3}\right) & \mathrm{s}\left(\theta_{2}\right) & -\mathrm{s}\left(2 \theta_{3}\right) \mathrm{c}\left(\theta_{2}\right) & -\mathrm{c} \theta_{2}\left[\mathrm{~d}_{4} \mathrm{c}\left(\theta_{3}\right)+\mathrm{d}_{7} \mathrm{~s}\left(2 \theta_{3}\right)\right] \\
\mathrm{s}\left(\theta_{2}\right) \mathrm{c}\left(2 \theta_{3}\right) & -\mathrm{c}\left(\theta_{2}\right) & -\mathrm{s}\left(2 \theta_{3}\right) \mathrm{s}\left(\theta_{2}\right) & -\mathrm{s} \theta_{2}\left[\mathrm{~d}_{4} \mathrm{c}\left(\theta_{3}\right)+\mathrm{d}_{7} \mathrm{~s}\left(2 \theta_{3}\right)\right] \\
-\mathrm{s}\left(2 \theta_{3}\right) & 0 & -\mathrm{c}\left(2 \theta_{3}\right) & \mathrm{d}_{1}+\mathrm{d}_{4} \mathrm{~s}\left(\theta_{3}\right)-\mathrm{d}_{7} \mathrm{c}\left(2 \theta_{3}\right) \\
0 & 0 & 0 & 1
\end{array}\right],
\end{aligned}
$$

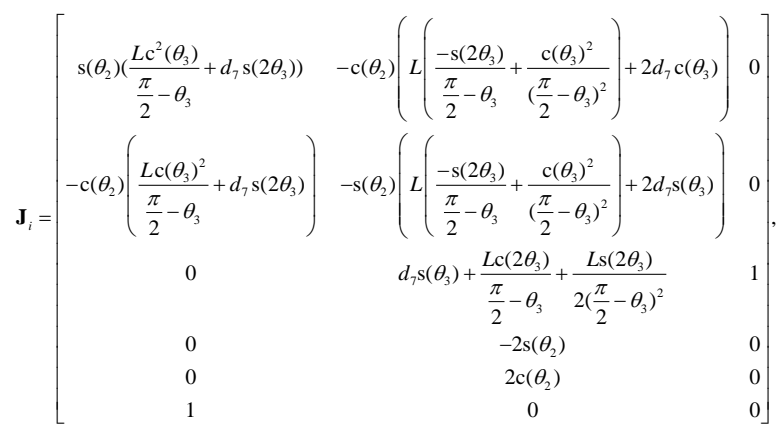

where $\mathrm{s}$ and $\mathrm{c}$ denote sin and cos functions, respectively. Notice that to obtain (8), the following geometry relation between $d_{4}$ and $\theta_{3}$ is used [25], 


$$
d_{4}=\frac{L}{\frac{\pi}{2}-\theta_{3}} \cos \theta_{3} .
$$

Therefore, the complete robot Jacobian $\mathbf{J}$ is obtained by stacking individual $\mathbf{J}_{i}$, i.e.,

$$
\mathbf{J}=\left(\begin{array}{llll}
\mathbf{J}_{1}{ }^{T} & \cdot & \cdot & \mathbf{J}_{n_{c}}{ }^{T}
\end{array}\right)^{T}
$$

where $n_{c}$ is the number of contact points, which equals 2 for the system shown in Fig. 4. Algorithm 1 shows the Jacobian formulation for Co-manipulative CCR.

Algorithm 1: Jacobian formation for co-manipulative CCR

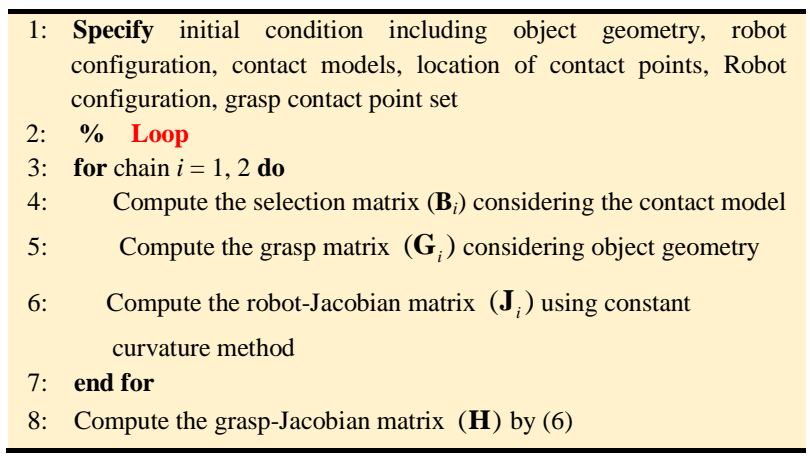

\section{TARGET-ASSIST CCRS}

Fig. 5 shows a set of two CRs operating in cooperation mode. A cooperation task could be broken down into the actuation of the distal end of the Target-CR and insertion of the assistive CR. These two actuations can occur simultaneously.

As illustrated in Part II, in the cooperation mode, insertion of the Assist-CR causes the tip of the Assist-CR applies forces and torques to the base of the bending section of the Target-CR which accordingly, helps the tip of the Target-CR reach a desired point outside its normal working space (Fig. 5-Right).

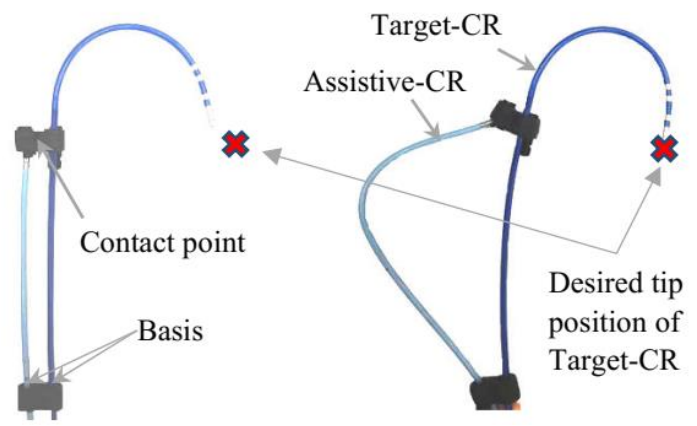

Figure 5. CCR in Cooperation Mode, (Left): The Target-CR is actuated, (Right): The Assist-CR is actuated to help the Target-CR reaches a desired point outside its reachability space.

In the sub-configuration shown in Fig. (5), interaction forces present at the contact point acting as external forces applied to each of Target- and Assist- CRs. Although this means that the system needs to be fully modeled using the Cosserat rod approach, similar to Part III, a deeper look into the characteristics of CRs, will enable us to use a simpler model. Two ends of Target-CR (i.e., the proximal and the distal ends) could be considered separately and accordingly modeled separately as well. The proximal end of Target-CR, along with Assistive-CR, forms a closed loop. In this part, the effect of the interaction forces and moments between the Target- and the Assis- CRs cannot be ignored since it causes displacement of the contact point and accordingly deformation of the corresponding parts of both CRs. Therefore, this part needs to be modeled using Cosserat rod.

On the other hand, the distal end of the Target-CR possesses one free end at the tip and one moving end at the contact point, which its movement comes from the displacement of the contact point as a result of the interaction forces and moments discussed in the previous paragraph. Therefore, kinematics of the distal end of the Target-CR could be modeled using a constant curvature assumption and DH-approach in which the base position of the distal end (the pose of the contact point) is updated by the Cosserat rod. Fig. 6 shows a schematic diagram of the proposed kinematic model.

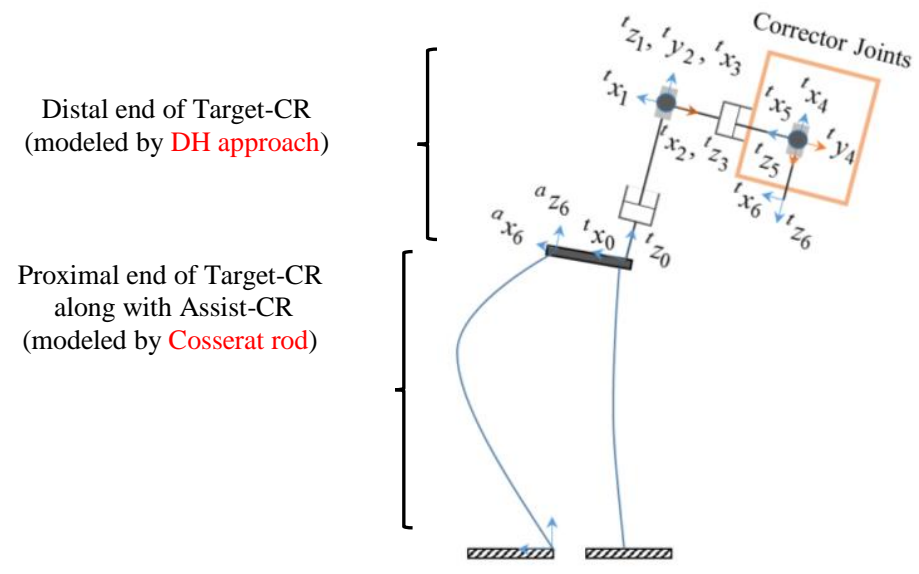

Figure 6. CCR in Cooperation mode: the assigned D-H parameters.

The pose of the target tip ( $\mathrm{T}_{\text {tip }}$ ) with.respect.to (w.r.t) the World frame located at the base of Target-CR can be obtained as

$$
{ }^{\mathrm{B}_{T} \mathrm{w}} \mathbf{H}_{\text {Ttip }}={ }^{\mathrm{B}_{T} \mathrm{~W}} \mathbf{H}_{\mathrm{CP}}\left({ }^{\mathrm{CPW}} \mathbf{H}_{\mathrm{CP}}\right)^{-1 \mathrm{CPW}} \mathbf{H}_{\text {Ttip }}
$$

where ${ }^{\mathrm{B}_{T} \mathrm{~W}} \mathbf{H}_{\mathrm{Ttip}},{ }^{\mathrm{B}_{T} \mathrm{w}} \mathbf{H}_{\mathrm{CP}},{ }^{\mathrm{CPW}} \mathbf{H}_{\mathrm{CP}}$, and ${ }^{\mathrm{CPW}} \mathbf{H}_{\text {Ttip }}$ are defined as,

${ }^{\mathrm{CPW}} \mathbf{H}_{\text {Ttip }}$ : The pose of $\mathrm{T}_{\text {tip }}$ (a frame located at the tip of the Target-CR) w.r.t CPW frame (a frame located at the contact point parallel to the World frame

${ }^{\mathrm{CPW}} \mathbf{H}_{\mathrm{CP}}$ : the orientation of the contact point frame $\{\mathrm{CP}\}$ w.r.t the world frame. The frame $\{\mathrm{CP}\}$ is a frame located at the contact point, the translation vector is zero, and the rotation matrix includes the rotation of contact point frame w.r.t the World frame.

${ }^{\mathrm{B}_{t} \mathrm{~W}} \mathbf{H}_{\mathrm{CP}}$ : the pose of the contact point w.r.t the World frame located at the base of Target-CR $\left\{\mathrm{B}_{\mathrm{t}} \mathrm{W}\right\}$.

Calculation of ${ }^{\mathrm{CPW}} \mathbf{H}_{\text {Ttip }}$ has a similar procedure as that discussed in Part III for the Co-manipulative sub-configuration. To obtain ${ }^{\mathrm{B}_{T} \mathrm{~W}} \mathbf{H}_{\mathrm{CP}}$, the Cosserat rod model needs to be utilized. Similar to 
[26], the following sets of equations defines the Cosserat rodbased model of a single CR:

Kinematics:
$\partial_{s} \mathbf{p}=\mathbf{R} \mathbf{v}$,
$\partial_{s} \mathbf{R}=\mathbf{\kappa} \times \mathbf{R}$.
Constitutive Relations:
$\mathbf{n}(s)=\mathbf{K}_{n e}\left(v-v^{0}\right)$
$\mathbf{m}(s)=\mathbf{K}_{m e}\left(\kappa-\kappa^{0}\right)$
Static Equations:
$\partial_{s} \mathbf{n}(s)=-\mathbf{\kappa} \times \mathbf{n}-\mathbf{R}^{\mathrm{T}} \mathbf{F}_{e x}$
$\partial_{s} \mathbf{m}(s)=-\mathbf{\kappa} \times \mathbf{m}-\mathbf{v}(s) \times \mathbf{n}-\mathbf{R}^{\mathrm{T}} \mathbf{L}_{e x}$
where
$\mathbf{K}_{m e}=\operatorname{diag}\left(E I_{1}, E I_{2}, G I_{3}\right), \mathbf{K}_{n e}=\operatorname{diag}(G A, G A, E A)$.

where in kinematic relations $\mathbf{p}, \mathbf{R}, \mathbf{v}$, and $\boldsymbol{\kappa}$ are position, orientation, linear strains, and angular strains, respectively. In constitutive relations, $\mathbf{n}(s)$ and $\mathbf{m}(s)$ represent internal forces and moments, and in the static equation, $\mathbf{F}_{e x}$ and $\mathbf{L}_{e x}$ stand for external forces and moments. Finally, $E, I_{i}, G$, and $A$ represent Young's modulus, the moment of inertia about axis $\{i\}$, shear modulus, and cross-section area.

A backward-forward iterative algorithm (Algorithm (2)) is employed to solve for the Equation-set (12). As the name suggests, the algorithm consists of two loops, the first loop accounts for calculating the strains (curvatures), and based on the obtained curvatures, the second loop calculates the orientation and position of the centroid curve of the CR.

Modeling of a set of two CRs has one more unknown parameter, which is the interaction forces and torques. A numerical algorithm could be used to find the interaction forces and torques so that when applying to the both CRs (i.e., the Assist-CR and the Target-CR), the contact pose will be obtained the same (Algorithm 3).

Finally, using the differential of the actuation input and the Target-CR tip positions, the Jacobian can be computed numerically for the closed-loop chain of the CCR system.

Algorithm 2. The algorithm to solve for kinematics of a single CR

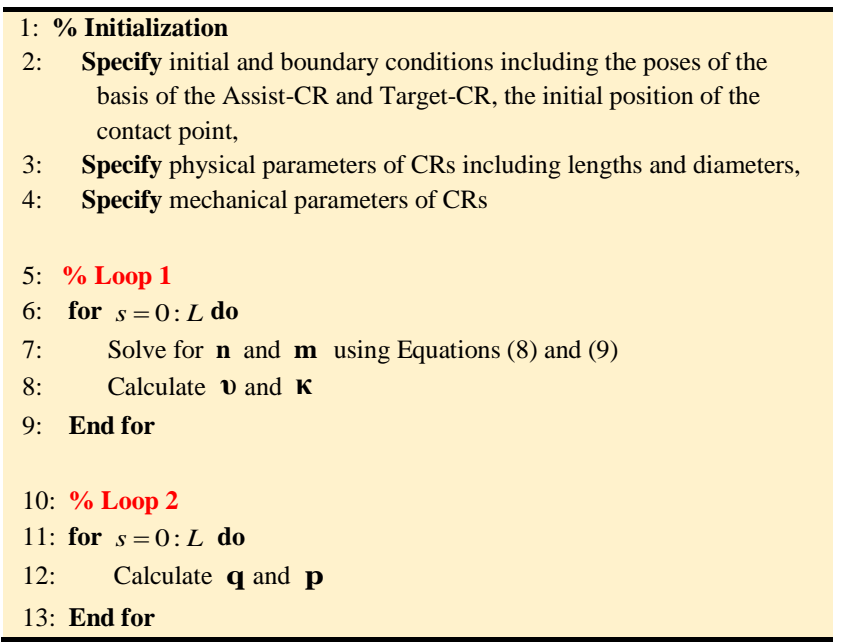

Algorithm 3. The pseudo-code to solve for kineto-statics behavior of a cooperative set of CRs

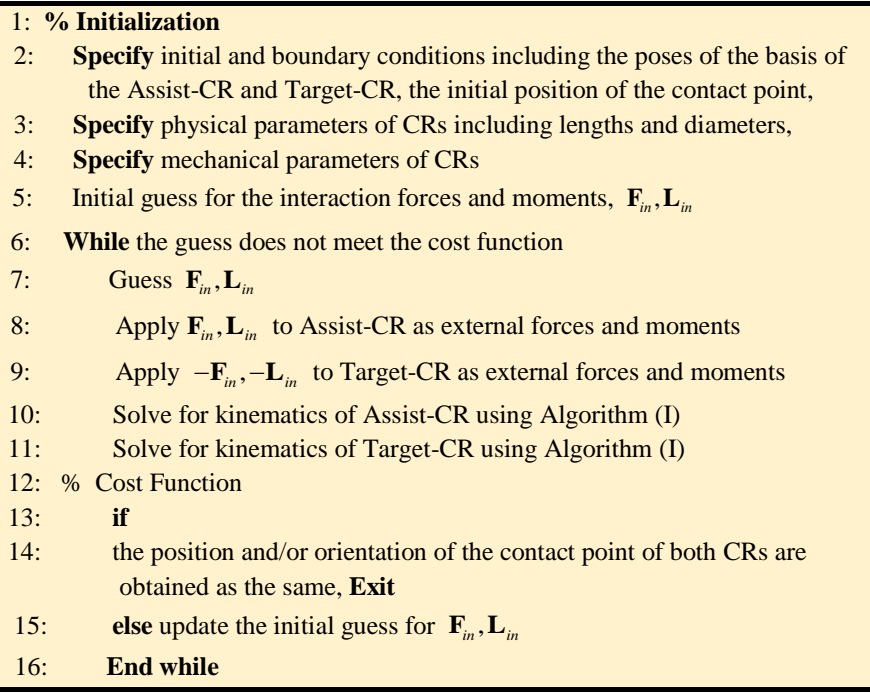

\section{IMPLEMENTATION}

In order to illustrate the performance of the algorithms presented in the previous sections, kinematic modeling of both sub-configurations of CCR is shown in Fig. 7. For this purpose, the structural information of a Bi-directional handle FlexAbility ${ }^{\mathrm{TM}}$ ablation catheters (St. Jude Medical, St. Paul, $\mathrm{MN}$ ) have been used.
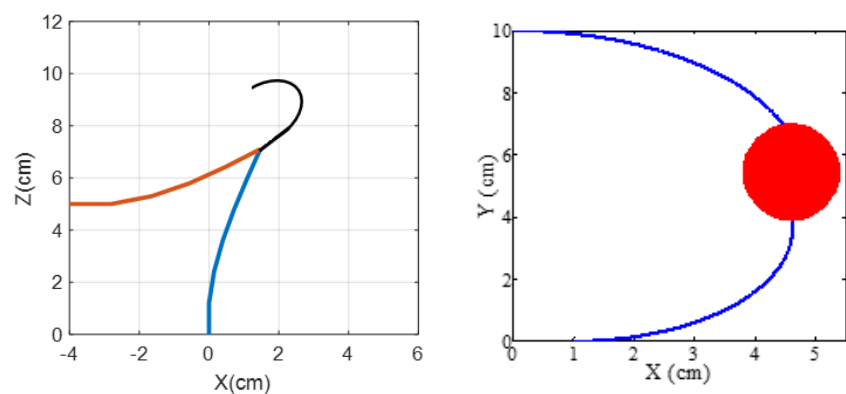

Figure 7. Sample simulation for Target-Assist and Co-manipulative subconfigurations

\section{CONCOLUSION}

This paper presented the kinematic analysis and Jacobian formulation of a new paradigm of continuum robots, entitles cooperative continuum robots (CCRs). First, a detailed discussion on the concept of CCR and its main subconfigurations is provided. Examples of such configurations are presented by a single segment tendon-driven catheter. Then, it extended previous works on the generation of Jacobians of CRs to the context of CCRs. Cosserat-rod and constant curvature approaches have been utilized for system modeling. The final Jacobians are relatively simple and applicable for a wide range of applications, from grasp synthesis to real-time control. Finally, a modeling example for a dual CCR is summarized.

\section{ACKNOWLEDGMENT}

This work was sponsored by the National Sciences and Engineering Research Council of Canada (NSERC) through 
Discovery Grant \# 2017-06930. The authors would also like to acknowledge the assistance of Dr. A. Cheema of St. Michael's Hospital (Toronto, ON) in clinical aspects of the work.

\section{REFERENCES}

[1] S. H. Sadati, S. E. Naghibi, A. Shiva, I. D. Walker, A. Kaspar, and T. Nanayakkara, "Mechanics of continuum manipulators, a comparative study of five methods with experiments," in Proc. Annu. Conf. Towar. Auton. Robot. Syst, 2017, pp. 686-702, Guildford, United Kingdom.

[2] J. D. Greer, T. K. Morimoto, A. M. Okamura, and E. W. Hawkes, "Series pneumatic artificial muscles ( sPAMs ) and application to a soft continuum robot," in Proc. IEEE Int. Conf. Robot. Autom., 2017, pp. 5503-5510, Singapore.

[3] G. Wu, G. Shi, and Y. Shi, "Modeling and analysis of a parallel continuum robot using artificial neural network," in Proc. IEEE Int. Conf. Mechatronics (ICM), 2017, pp. 153-158, Australia.

[4] A. Lotfavar, S. Hasanzadeh, and F. Janabi-Sharifi, "Cooperative continuum robots: concept, modeling, workspace analysis," IEEE Robot. Autom. Lett., vol. 3, no. 1, pp. 426-433, 2017.

[5] S. H. Sadati, S. Naghibi, I. D. Walker, K. Althoefer, and T. Nanayakkara, "Control space reduction and real-time accurate modeling of continuum manipulators using Ritz and Ritz-galerkin methods," IEEE Robot. Autom. Lett., vol. 3, no. 1, pp. 328-335, 2018.

[6] C. Bryson Black, "Modeling, Analysis, Force Sensing and Control of Continuum Robots for Minimally Invasive Surgery," $\mathrm{PhD}$ diss, University of Tennessee, 2017.

[7] F. Renda, M. Giorelli, M. Calisti, M. Cianchetti, and C. Laschi, "Dynamic model of a multibending soft robot arm driven by cables," IEEE Trans. Robot., vol. 30, no. 5, pp. 1109-1122, 2014.

[8] M. W. Hannan and I. D. Walker, "Kinematics and the implementation of an elephant's trunk manipulator and other continuum style robots," $J$. Robot. Syst., vol. 20, no. 2, pp. 45-63, 2003.

[9] M. Calisti et al., "An octopus-bioinspired solution to movement and manipulation for soft robots," Bioinspir. Biomim., vol. 6, no. 3, pp. 36002-36012, 2011.

[10] R. J. Webster and B. a. Jones, "Design and kinematic modeling of constant curvature continuum robots: A review," Int. J. Rob. Res., vol. 29, no. 13, pp. 1661-1683, 2010.

[11] I. S. Godage, D. T. Branson, E. Guglielmino, G. A. Medrano-Cerda, and D. G. Caldwell, "Shape function-based kinematics and dynamics for variable length continuum robotic arms," in Proc. IEEE Int. Conf. Robot. Autom., 2011, pp. 452-457, Shanghai, China.

[12] A. Kapadia and I. D. Walker, "Task-space control of extensible continuum manipulators," IEEE/RSJ Int. Conf. Intell. Robot. Sys., pp.
1087-1092, San Francisco, California, USA., 2011.

[13] I. D. Walker et al., "Continuum robot arms inspired by cephalopods," Unmanned Gr. Veh. Tech. VII, vol. 5804, pp. 303-314, International Society for Optics and Phot, 2005.

[14] W. S. Rone and P. Ben-Tzvi, "Continuum robot dynamics utilizing the principle of virtual power," IEEE Trans. Robot., vol. 30, no. 1, pp. 275287, 2014.

[15] R. Kang, D. T. Branson, E. Guglielmino, and D. G. Caldwell, "Dynamic modeling and control of an octopus inspired multiple continuum arm robot," Comput. Math. with Appl., vol. 64, no. 5, pp. 1004-1016, 2012.

[16] C. Della Santina, R. K. Katzschmann, A. Bicchi, and D. Rus, "Dynamic control of soft robots interacting with the environment," in Proc. IEEE Int. Conf. on Soft Robot., RoboSoft 2018, 2018, pp. 46-53, Livorno, Italy.

[17] M. Z. Benjamin Conrad, "Closed loop task space control of an interleaved continuum rigid manipulator," in Proc. IEEE Int. Conf. Robot. Autom., 2015, pp. 1743-1750, Washington, USA.

[18] N. Simaan, R. Taylor, and P. Flint, "A dexterous system for laryngeal surgery," IEEE Int. Conf. Robot. Autom. 2004. Proceedings. ICRA '04. 2004, vol. 1, no. April, pp. 351-357, 2004.

[19] K. Xu, M. Fu, and J. Zhao, "An experimental kinestatic comparison between continuum manipulators with structural variations," in Proc. IEEE Int. Conf. Robot. Autom., 2014, pp. 3258-3264, Hong Kong, China.

[20] J. Till, C. E. Bryson, S. Chung, A. Orekhov, and D. C. Rucker, "Efficient computation of multiple coupled Cosserat rod models for real-time simulation and control of parallel continuum manipulators," Proc. IEEE Int. Conf. Robot. Autom., vol. 2015-June, no. June, pp. 5067-5074, 2015.

[21] J. Till and D. C. Rucker, "Elastic stability of cosserat rods and parallel continuum robots," IEEE Trans. Robot., vol. 33, no. 3, pp. 718-733, 2017.

[22] J. Burgner-Kahrs, D. C. Rucker, and H. Choset, "Continuum robots for medical applications: A survey," IEEE Trans. Robot., vol. 31, no. 6, pp. 1261-1280, 2015.

[23] D. Rus and M. T. Tolley, "Design, fabrication and control of soft robots," Nature, vol. 521, no. 7553, pp. 467-475, 2015.

[24] B. León, A. Morales, and J. Sancho-Bru, From Robot to Human Grasping Simulation, vol. 19. 2014.

[25] I. D. Walker, "Continuous backbone 'continuum' robot manipulators," ISRN Robot., pp. 1-19, 2013.

[26] R. J. Webster III and D. C. Rucker, "Statics and dynamics of continuum robots with general tendon routing and external loading," IEEE Trans. Robot., vol. 27, no. 6, pp. 1033-1044, 2011. 